\title{
Evaluation of CXCL10 as a novel serum marker for predicting liver metastasis and prognosis in colorectal cancer
}

\author{
YUJI TOIYAMA, HIROYUKI FUJIKAWA, MIKIO KAWAMURA, KOHEI MATSUSHITA, SUSUMU SAIGUSA, \\ KOJI TANAKA, YASUHIRO INOUE, KEIICHI UCHIDA, YASUHIKO MOHRI and MASATO KUSUNOKI
}

Department of Gastrointestinal and Pediatric Surgery, Mie University Graduate School of Medicine, Tsu, Mie 514-8507, Japan

Received August 2, 2011; Accepted September 21, 2011

DOI: 10.3892/ijo.2011.1247

\begin{abstract}
The aim of this study was to identify novel and reliable serum markers related to the prognosis of colorectal cancer (CRC) patients and to assess the association between selected markers and clinical outcome. We performed experiments using cytokine arrays to investigate the cytokine profiles in serum from stage IV CRC patients, compared with those of stage I patients. Serum CXCL10 was measured using an ELISA in 218 CRC patients and 17 normal volunteers to clarify the association of CXCL10 with clinical outcome. The mean serum CXCL10 concentration in CRC patients was significantly higher compared to that in normal volunteers. Serum CXCL10 levels increased significantly in accordance with the progression of UICC stage classification. Serum CXCL10 was significantly associated with high pathological $\mathrm{T}$ stage, the presence of vascular invasion and distant metastasis. Elevated serum CXCL10 levels were significantly associated with poor survival in all stages or in stage I-III with curative patients, respectively, and were an independent marker in predicting liver metastasis. Immunohistochemical analysis showed that CXCL10 was expressed in cancer cells at primary tumor and liver metastases sites, and in normal liver tissue surrounding metastatic cancer cells. Comprehensive analysis using cytokine arrays identified the novel serum prognosis marker CXCL10. Preoperative high serum levels of CXCL10 were associated with poor prognosis and liver metastasis in CRC.
\end{abstract}

\section{Introduction}

Colorectal cancer (CRC) is the third most common cancer in the world and the fourth most common cause of cancer related death (1). Survival rates of patients with CRC have increased in the past few years, possibly as a result of early diagnosis and improved treatment. However, approximately $60 \%$ of patients

Correspondence to: Dr Yuji Toiyama, Department of Gastrointestinal and Pediatric Surgery, Mie University Graduate School of Medicine, 2-174 Edobashi, Tsu, Mie 514-8507, Japan

E-mail: ytoi0725@clin.medic.mie-u.ac.jp

Key words: colorectal cancer, CXCL10, prognostic marker, liver metastasis who undergo curative resection experience local recurrence or distant metastases $(2,3)$. Patients with local recurrence or distant metastases usually receive chemotherapy in combination with monoclonal antibody therapy, and the median overall survival time is around 20-21 months, with a response rate of around $50 \%$ (4). However, almost half the patients experience treatment-related side effects, such as diarrhea, mouth irritation, and low white blood cell count, with no therapeutic benefit. The identification of CRC patients at high risk of metastasis would thus aid in the selection of appropriate candidates for standard or intense adjuvant therapy.

In general, serum tumor markers can be an easy-to-use approach for the detection of cancer, and carcinoembryonic antigen (CEA) is the marker most frequently evaluated as a predictor of prognosis in patients with CRC. However, serum CEA levels do not correlate with the presence of metastasis, and the incidences of false-positive and false-negative results are high $(5,6)$. We therefore, attempted to identify novel and reliable serum markers for prognosis and distant metastasis using a human cytokine antibody array capable of analyzing 174 cytokines in human serum. By comparing serum cytokine expression in serum between patients with stage IV and stage I CRC, we found that CXC chemokine ligand 10 (CXCL10) was markedly upregulated in stage IV patients.

Chemokines are a large family of small chemotactic cytokines with four conserved cysteines linked by disulfide bonds. Two subfamilies, the CC and CXC chemokines, can be distinguished according to the position of the first two cysteine residues, which are either adjacent to each other or separated by one amino acid. CXC chemokines are important for enhancing immunity, regulating angiogenesis, and mediating tumor cell metastases (7). CXCL10, human interferon-inducible protein 10 (IP-10), acts through putative receptor CXC receptor 3 (CXCR3), and functions as a major chemoattractant for activated $\mathrm{T}$ cells and natural killer cells (8-10). Recently, numerous studies into the role of CXCL10 in malignancy have shown two tumor inhibiting properties that influence cells in the tumor microenvironment (11). One property is the recruitment into tumor sites of CXCR3-expressing mononuclear cells with antitumor activity and the other is antagonizing the effects of powerful angiogenic factors (8-12).

Although the antimalignancy activities of local CXCL10 expression were determined in several tumor systems, only a limited number of studies, primarily murine models, analyzed 
the role of CXCL10 in CRC (13-17). Moreover, we believe that the clinicopathological significance of CXCL10 has not been fully studied in clinical serum samples of CRC. Thus, in this retrospective study, we investigated CXCL10 expression in preoperative serum samples from CRC patients to determine any clinicopathological and prognostic significance.

\section{Materials and methods}

Cytokine array analysis of patient serum. Preoperative serum was collected from five patients with stage IV or stage I CRC who underwent resection at our institution between January 1996 and January 2009. Sera from patients with the same stage tumors were mixed, and 174 cytokines were analyzed using a human cytokine antibody array (G Series 2000, RayBiotech, Norcross, GA, USA), according to the manufacturer's instructions. Briefly, blocking buffer was added into each well of the microarray glass slides for $30 \mathrm{~min}$. Samples (100 $\mu \mathrm{l}$ per well) were added and incubated at room temperature for $2 \mathrm{~h}$. The slides were washed in washing buffer and incubated with a biotin-conjugated cocktail of antibodies at room temperature for $2 \mathrm{~h}$. After further washing, slides were incubated with 1:1,500 diluted fluorescent dye-conjugated streptavidin at room temperature in darkness for $2 \mathrm{~h}$. Fluorescent signal was detected using a confocal laser scanner (HB GeneArray Scanner, Affymetrix) and analyzed using the RayBio Antibody Array Analysis tool (RayBiotech).

Patients and sample collection. A total of 218 consecutive patients (145 men and 73 women; mean age 64.7 years; age range 12-88 years) who underwent resection of CRC at our institution between January 1996 and January 2009, and for whom preoperative serum could be collected, were enrolled in this retrospective study. No preoperative mortality was observed among the patients. Patients with inflammatory diseases, including infectious or collagen diseases, were excluded from the study. All patients were classified according to UICC stage classifications using resected specimens. There were 47 patients with stage I disease, 57 patients with stage II disease and 59 patients with stage III disease. Fifty-five patients with distant metastases were classified as having stage IV disease. The median follow-up time was 48 months (range 2-107 months). The control group consisted of 20 age- and gender-matched healthy volunteers. The absence of disease was assessed on the basis of clinical history, physical examination, and routine laboratory tests, including liver and renal function tests.

Blood samples were obtained by venipuncture before surgery. Each sample was centrifuged at $3,000 \mathrm{~g}$ for $5 \mathrm{~min}$ and then frozen at $-80^{\circ} \mathrm{C}$ until analysis. Written informed consent for the use of the samples in future experiments was obtained from all participants before serum collection. All investigations were performed in accordance with the Helsinki Declaration and were approved by our Institutional Review Board.

Determination of serum CXCL10 level in CRC patients and normal volunteers. Serum CXCL10 levels were determined using an enzyme-linked immunosorbent assay (ELISA) kit (Human IP-10 ELISA kit, Invitrogen, Carlsbad, CA), according to the manufacturer's instructions. Samples $(50 \mu \mathrm{l})$ were incubated in microtiter plates pre-coated with a polyclonal antibody specific for CXCL10. After incubation at room temperature for $2 \mathrm{~h}$ and washing, CXCL10 conjugate was added. The substrate solution was added after a further 2-h incubation at room temperature and washing. Color development was stopped after $30 \mathrm{~min}$ at room temperature, and the intensity was read at $450 \mathrm{~nm}$ within $30 \mathrm{~min}$. Results were calculated from a standard curve (recombinant human CXCL10; range 7.8-500 ng/ml) generated from a four-parameter logistic curve fit.

Immunohistochemical analysis of CXCL10 in primary and metastatic CRC sites. Formalin-fixed, paraffin-embedded specimens were cut into $2-\mu \mathrm{m}$-thick sections. After deparaffinization and dehydration, antigen unmasking was performed by boiling in $10 \mathrm{mM}$ sodium citrate buffer. Specimens were then blocked and incubated with primary antibody overnight at $4^{\circ} \mathrm{C}$. The antibody was detected using Envision reagents (Envision kit/HRP, Dako Cytomation, Denmark). All sections were counterstained with hematoxylin. Primary anti-CXCL10 goat polyclonal antibody (clone AF976, R\&D Systems, MN, USA) and anti-CXCR3 rabbit polyclonal antibody (clone ab8023, Abcam, Cambridge, UK) at a dilution of 1:25 were used for the labeled streptavidinbiotin method (LASB2 kit/HRP, Dako Cytomation). Negative controls using preimmune immunoglobulin were run simultaneously.

Statistical analyses. Data are expressed as median values (interquartile range) in tables, or as means \pm standard error (SE) in figures. Comparisons were performed using the non-parametric Mann-Whitney U-test for continuous variables. Analyses of non-parametric receiver operating characteristics (ROC) were performed, using JMP 7 for Windows software (SAS Institute, Cary, NC), to calculate the cut-off values, according to the most accurate value obtained in all CRC patients. Survival probabilities were calculated using the product limit of the Kaplan-Meier method, considering treatment- and CRC-related deaths. Differences between two groups were determined using the log-rank test. Logistic regression analysis was used to evaluate the independent influence of factors on liver metastasis. All statistical analyses were carried out using StatView 5.0 for Windows (SAS Institute, Inc.). Two-tailed p-values $<0.05$ were considered statistically significant.

\section{Results}

Elevated serum cytokine profiles in stage IV CRC patients. We initially performed cytokine array analysis to investigate the cytokine profiles in serum from stage IV CRC patients $(n=5)$, compared with those of stage I patients (Fig. 1a). Seven cytokines (CXCL10, IL-15, ENA-78, IL-18 Bp $\alpha$, CXCL16, TGF- $\beta 1$, MCP-4) were elevated 2-fold in stage IV CRC serum samples compared with stage I samples (Fig. 1b). Serum CXCL10 was elevated 2.2-fold in stage IV CRC serum samples compared with stage I CRC serum samples.

Associations between serum CXCL10 and clinicopathological findings in CRC patients. Cytokine array analysis identified serum CXCL10 levels to be significantly elevated in patients with stage IV CRC. To confirm the association between serum CXCL10 and clinicopathological findings, serum CXCL10 levels were analyzed in $218 \mathrm{CRC}$ patients and 17 normal 
a
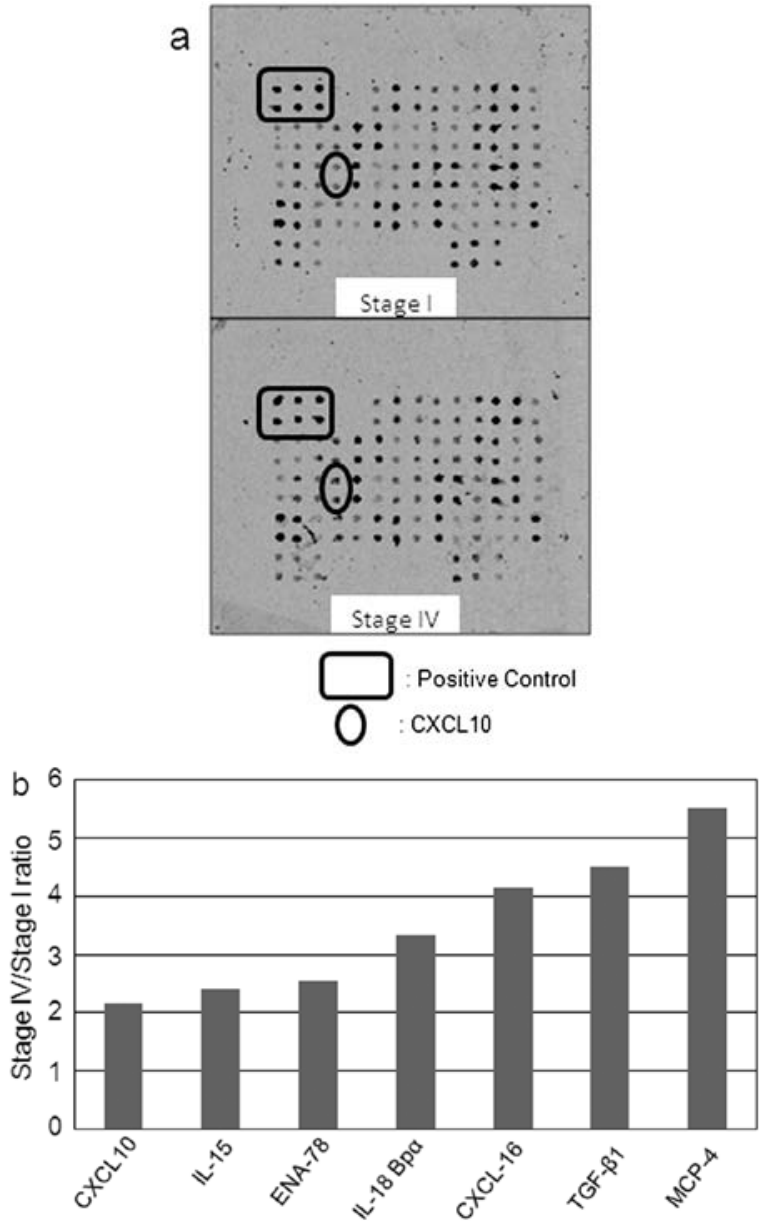

Figure 1. (a), Cytokine antibody array analysis was used to investigate serum cytokine profiles in patients with stage IV colorectal cancer (CRC), compared with patients with stage I CRC. (b), The vertical axis shows the relative expression ratios of several cytokines in stage IV patients, compared with those in stage I patients. controls using ELISA. The serum concentrations of CXCL10 in patients ranged from 24 to $933 \mathrm{pg} / \mathrm{ml}$. By contrast, the serum concentrations of CXCL10 in normal volunteers ranged from 42 to $269 \mathrm{pg} / \mathrm{ml}$. The mean serum CXCL10 concentration in patients was significantly higher than that in normal volunteers (162 vs. $103 \mathrm{pg} / \mathrm{ml}$; p<0.05; Fig. 2). In addition, serum CXCL10 levels increased significantly in accordance with the progression of UICC stage classification $(\mathrm{p}<0.05$; Fig. 2). Table I shows the relationship between serum CXCL10 levels and clinicopathological findings. High serum CXCL10 was associated with being aged over 67 years $(\mathrm{p}=0.0004)$, high pathological T stage such as T3 or T4 $(\mathrm{p}=0.0013)$, the presence of vascular invasion $(\mathrm{p}=0.0068)$, liver metastases $(\mathrm{p}=0.0013)$ and distant metastases $(\mathrm{p}=0.0003)$.

In particular, serum CXCL10 level increased significantly in accordance to pathological T stage (Fig. 2b) and clinical $\mathrm{H}$ stage (Fig. 2c). Furthermore, a significant correlation was found between serum CXCL10 and serum CEA (Fig. 2d).

Associations between serum CXCL10 and survival in CRC. To examine the predictive value of serum CXCL10 for prognosis in CRC patients, we defined the cut-off value according to the best predictive values calculated by ROC analysis (cut-off value $=$ $199 \mathrm{pg} / \mathrm{ml}$ ). Higher serum CXCL10 level was associated with a poor prognosis ( $\mathrm{p}=0.002$, log-rank test; Fig. $3 \mathrm{a}$ ). On the basis of Cox univariate proportional hazards analysis, pathological T stage (T3, T4; $p<0.0001$ ), CEA ( $\geq 6 \mathrm{ng} / \mathrm{ml} ; \mathrm{p}<0.0001$ ), lymph node metastasis $(\mathrm{p}=0.0001)$, vessel involvement $(\mathrm{p}=0.0001)$, lymphatic vessel involvement $(\mathrm{p}=0.0062)$, distant metastasis $(p<0.0001)$ and elevated serum CXCL10 level $(p=0.0002)$ were significant prognostic factors for cancer specific survival. Multivariate analysis identified lymph node metastasis ( $\mathrm{p}=0.03$ ), elevated serum CXCL10 level and distant metastasis as independent risk factors for predicting poor prognosis (Table II).
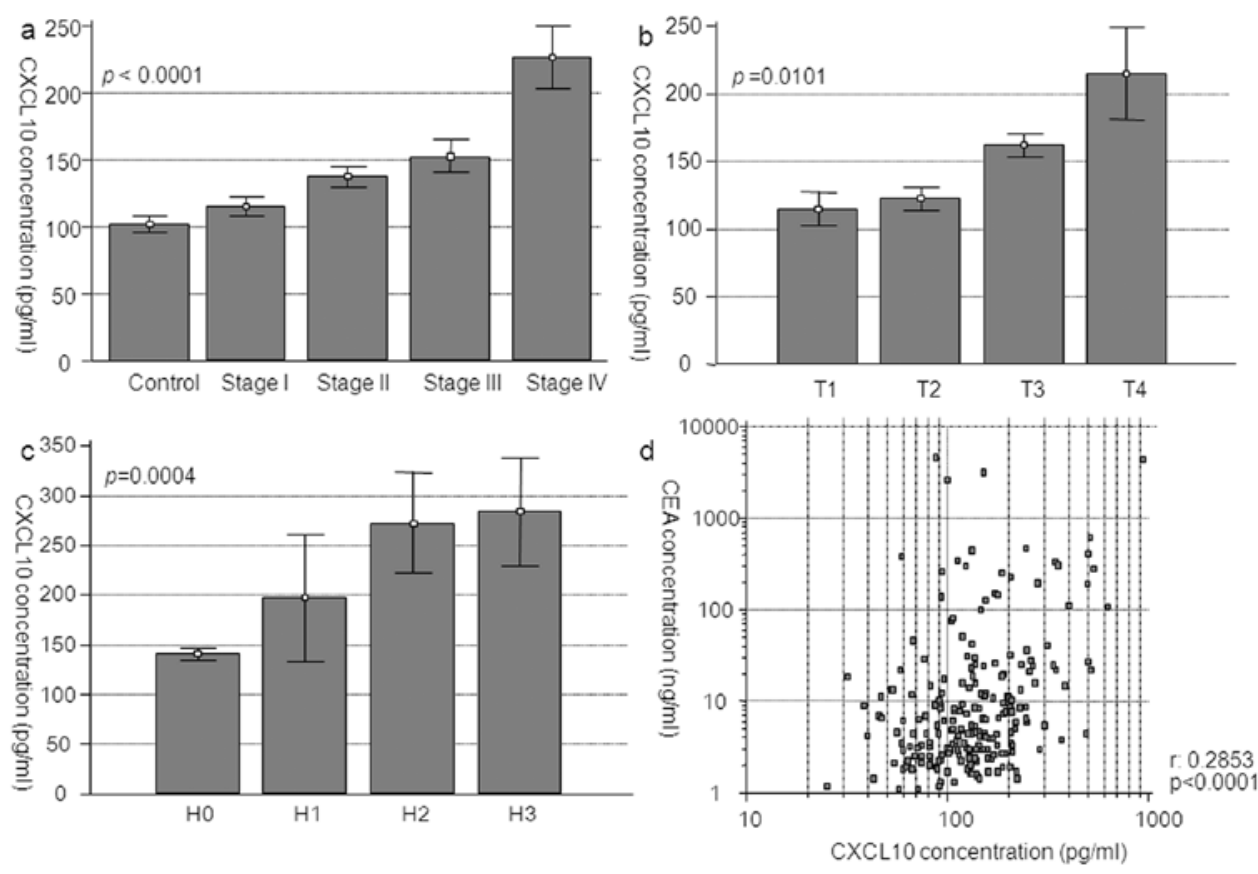

Figure 2. (a), Scattergram of serum CXCL10 in stage I-IV colorectal cancer patients and control subjects ( $<<0.0001)$. (b), Scattergram of serum CXCL10 subdivided by pathological T stage ( $\mathrm{p}=0.0101)$. (c), Scattergram of serum CXCL10 subdivided by clinical H stage ( $\mathrm{p}=0.0004)$. (d), The relationships between serum CXCL10 and serum CEA level $(\mathrm{r}=0.2853, \mathrm{p}<0.0001)$. 
Table I. Relationships between serum CXCL10 and clinicopathological factors in colorectal cancer patients.

\begin{tabular}{|c|c|c|c|}
\hline Variables & $\begin{array}{l}\text { No. of } \\
\text { patients }\end{array}$ & $\begin{array}{c}\text { CXCL10 } \\
\text { (mean) }\end{array}$ & P-value \\
\hline \multicolumn{4}{|l|}{ Age (year) } \\
\hline$<67$ & 103 & 140.43 & \\
\hline$\geq 67$ & 115 & 175.6 & 0.0004 \\
\hline
\end{tabular}

Gender

$\begin{array}{lrrr}\text { Female } & 73 & 159.6 & \\ \text { Male } & 145 & 158.5 & 0.73\end{array}$

Vascular invasion

$\begin{array}{lrrr}\text { No } & 63 & 124.8 & \\ \text { Yes } & 155 & 172.8 & 0.0068\end{array}$

Lymphatic invasion

$\begin{array}{lrrr}\text { No } & 34 & 133.9 & \\ \text { Yes } & 184 & 163.5 & 0.0635 \\ & & & \\ \text { athological N } & & & \\ \text { No } & 123 & 139.9 & \\ \text { Yes } & 95 & 180.9 & 0.1704\end{array}$

Pathological T

$\begin{array}{rrrr}\mathrm{T} 1.2 & 59 & 119.5 & \\ \mathrm{~T} 3.4 & 159 & 172.2 & 0.0013\end{array}$

Pathology

Differentiated $\quad 198 \quad 158.62$

$\begin{array}{llll}\text { Non-differentiated } & 20 & 144.7 & 0.6069\end{array}$

Peritoneal dissemination

$\begin{array}{lrrr}\text { No } & 210 & 157.3 & \\ \text { Yes } & 8 & 199.8 & 0.8016\end{array}$

Hepatic metastasis

$\begin{array}{lrrr}\text { No } & 189 & 141.3 & \\ \text { Yes } & 29 & 253.5 & 0.0013\end{array}$

Distant metastasis (all)

\begin{tabular}{lrrr} 
No & 163 & 136.2 & \\
Yes & 55 & 224.5 & 0.0003 \\
\hline
\end{tabular}

Furthermore, to examine the predictive value of serum CXCL10 for the prognosis of 163 patients in stage I-III with curative intent, we defined the cut-off value according to the best predictive values calculated by ROC analysis (cut-off value $=153 \mathrm{pg} / \mathrm{ml}$ ). Kaplan-Meier analysis showed that higher serum CXCL10 level was associated with a poor prognosis $(\mathrm{p}=0.032, \log$-rank test; Fig. 3b).

Associations between serum CXCL10 and liver metastasis in CRC patients. Factors correlated with liver metastasis in CRC were analyzed by logistic regression. The predictive value of serum CXCL10 for liver metastasis was also examined by defining the cut-off value using ROC analysis (cut-off value = $203 \mathrm{pg} / \mathrm{ml}$ ). Significant clinical parameters for predicting liver metastasis were pathological T stage (T3, T4; $\mathrm{p}=0.0001)$, CEA $\geq 6 \mathrm{ng} / \mathrm{ml}(\mathrm{p}<0.0001)$, lymph node metastasis $(\mathrm{p}=0.004)$, vessel involvement $(p=0.014)$ and high serum CXCL10 levels $(\mathrm{p}<0.0001$; Table III). Multivariate logistic analysis identified high serum CXCL10 level as the most significant independent clinical parameter associated with liver metastasis (high serum CXCL10, $\mathrm{p}=0.0067$; CEA $\geq 6 \mathrm{ng} / \mathrm{ml}, \mathrm{p}=0.01$; Table III).

Expression patterns of CXCL10 in primary CRC and liver metastases. Fig. 4 shows representative immunostaining of cancerous regions in CRC tissues, normal mucosa, and liver metastasis tissues. CXCL10 was diffusely expressed in the cytoplasm of primary CRC, but barely detectable in the cancer stroma and normal mucosa. Cancerous regions in liver metastasis tissues stained for CXCL10 in the same way as primary CRC. In addition, CXCL10 was located diffusely in the cytoplasm in normal liver tissues (Fig. 4).

\section{Discussion}

Biomarkers play an important role in cancer diagnosis, prognosis, treatment, and monitoring. Serum biomarker detection has been widely applied for convenience and because it is minimally invasive. In recent years, with the development of molecular biological techniques and new discoveries in cancer biology, many serum biomarkers related to diagnosis, prognosis, and patient survival have been explored. In our study, we comprehensively analyzed serum markers related with prognosis in CRC by using cytokine array to consequently identify the novel prognostic marker CXCL10. We next reassessed prognostic significance of serum CXCL10 in 218 additional CRC patients. Cancer specific survival of patients with high CXCL10 was significantly poorer than that with low CXCL10 in stage I-III or stage I-IV, respectively. Furthermore, multivariate analysis showed that an elevated serum CXCL10 level was found to be an independent prognostic variable. These results suggested that cytokine array is a very useful method for detecting serum biomarkers and revealed CXCL10 as a novel prognostic marker in CRC patients.

Classically, interactions between CXCL10 and its receptor, CXCR3, play important roles in the pathogenesis of autoimmune and inflammatory diseases through the recruitment of $\mathrm{CXCR}^{+} \mathrm{T}$ cells (18-20). The recruitment of cytotoxic $\mathrm{T}$ cells induced via CXCL10-CXCR3 interactions could also facilitate antitumor immunity, resulting in tumor regression $(21,22)$. Thus, the overexpressed CXCL10 in human CRC also may attract $\mathrm{CXCR}^{+}{ }^{+} \mathrm{T}$ cells to the tumor and promote antitumor immunity (23-25). Clinically, CXCL10 has been characterized as a prognostic marker for predicting outcome in uterine cervical cancer and CRC $(26,27)$. In fact, lower expression of CXCL10 in cancer tissue using immunohistochemical analysis was linked with poor prognosis in these patients.

Recently, accumulating evidence has shown that chemokine interactions between tumor cells and their microenvironment are complex, with responses to chemokines ranging from growth support to inhibition of the tumorigenesis process (28). CXCL10 has been reported to be expressed in significant 

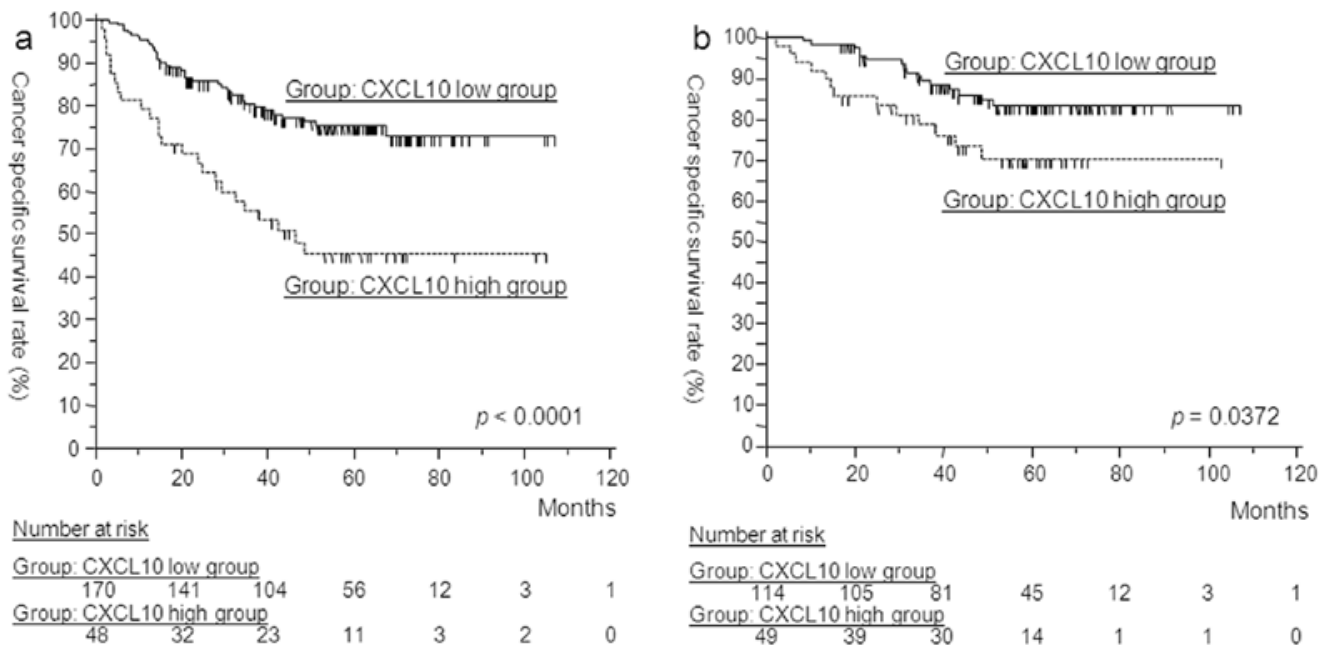

Number at risk

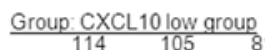

Group: $\mathrm{CXCL} 10$ high group

$\begin{array}{llll}45 & 12 & 3 & 1 \\ 14 & 1 & 1 & 0\end{array}$

Figure 3. (a), Cancer specific survival curves, for 218 patients with colorectal cancer, according to serum CXCL10 level. Patients with higher serum CXCL10 had significantly poorer prognoses than those with lower serum CXCL10 levels ( $<<0.0001, \log$-rank test); cut-off value $=199 \mathrm{pg} / \mathrm{ml}$. (b), Cancer specific survival curves for 163 patients in stage I-III with curative intent, according to serum CXCL10 level. Patients with higher serum CXCL10 also had significantly poorer prognoses than those with lower serum CXCL10 levels ( $\mathrm{p}=0.0372$, log-rank test); cut-off value $=153 \mathrm{pg} / \mathrm{ml}$.

Table II. Uni- and multivariate analyses for prognostic factors in colorectal cancer.

\begin{tabular}{lcccccc}
\hline Variables & OR & $95 \%$ CI & P-value & OR & $95 \%$ CI & P-value \\
\hline Age ( $\geq 67$ vs. $<67)$ & 0.95 & $0.58-1.54$ & 0.81 & - & - & - \\
Gender (female vs. male) & 0.91 & $0.54-1.54$ & 0.75 & - & - & - \\
Pathological T (T3.4 vs. T1.2) & 5.46 & $2.2-13.55$ & $<0.0001$ & 2.38 & $0.9-6.27$ & 0.08 \\
Vessel involvement (yes vs. no) & 3.88 & $1.77-8.48$ & 0.0001 & 1.73 & $0.76-3.91$ & 0.18 \\
Lymphatic vessel involvement (yes vs. no) & 3.27 & $1.19-8.95$ & 0.0062 & 1.31 & $0.45-3.73$ & 0.61 \\
Pathology (poor differentiation vs. differentiation) & 1.12 & $0.48-2.60$ & 0.78 & - & - & - \\
Pathological N (yes vs. no) & 2.68 & $1.61-4.47$ & 0.0001 & 1.72 & $1.02-2.88$ & 0.04 \\
Distant metastasis (yes vs. no) & 5.45 & $3.32-8.95$ & $<0.0001$ & 3.47 & $2.01-5.81$ & $<0.0001$ \\
CEA (>6 vs. <6) & 3.04 & $1.78-5.18$ & $<0.0001$ & 1.54 & $0.87-2.81$ & 0.13 \\
CXCL10 (high vs. low) & 2.75 & $1.67-4.54$ & 0.0002 & 1.6 & $1.01-2.68$ & 0.05 \\
\hline
\end{tabular}

Table III. Uni- and multivariate analyses for predicting liver metastasis in colorectal cancer.

\begin{tabular}{lccc}
\hline Variables & OR & $95 \%$ CI & P-value \\
\hline Univariate regression analysis & & & 0.0001 \\
Pathological T (T3.4 vs. T1.2) & 14.57 & $1.88-106.2$ & 0.0006 \\
Vessel involvement (yes vs. no) & 7.37 & $1.70-31.89$ & 0.2625 \\
Lymphatic vessel involvement (yes vs. no) & 1.94 & $0.55-6.79$ & 0.41 \\
Pathology (poor differentiation vs. differentiation) & 1.67 & $0.52-5.34$ & 0.0005 \\
Pathological N (yes vs. no) & 4.01 & $1.75-9.15$ & $<0.0001$ \\
CEA (>6 vs. $<6$ ) & 5.81 & $2.28-14.79$ & $<0.0001$ \\
CXCL10 (high vs. low) & 5.37 & $2.42-11.95$ & 0.14 \\
\hline Multivariate regression analysis & & & 0.09 \\
Pathological T (T3.4 vs. T1.2) & 4.77 & $0.59-38.46$ & 0.06 \\
Vessel involvement (yes vs. no) & 3.84 & $0.79-18.56$ & 0.016 \\
Pathological N (yes vs. no) & 2.234 & $0.95-5.77$ & 0.002 \\
CEA (>6 vs. $<6$ ) & 3.36 & $1.24-9.11$ & $1.65-9.46$ \\
CXCL10 (high vs. low) & 3.94 & & \\
\hline
\end{tabular}



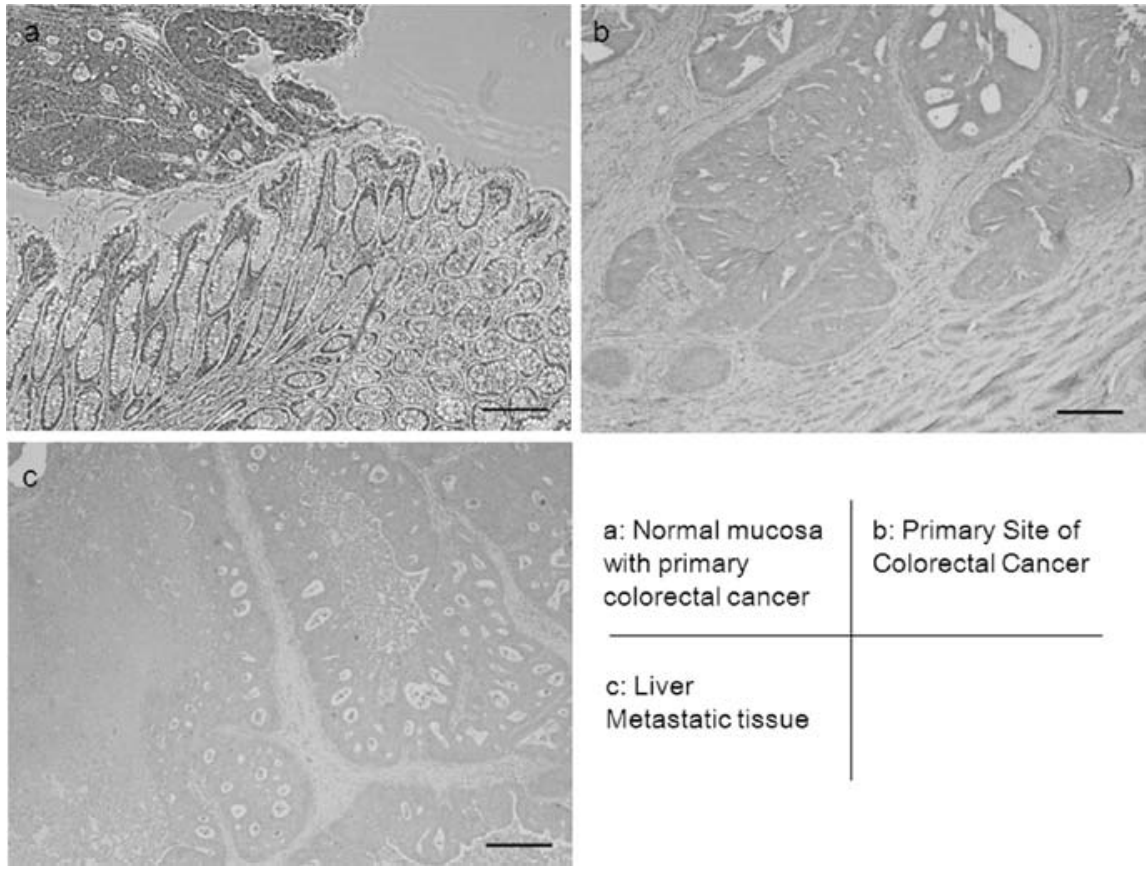

Figure 4. CXCL10 protein expression was assessed using immunohistochemistry in normal mucosa with primary CRC (a), primary colorectal cancer (b) and liver metastasis tissue (c). Magnification, $\mathrm{x} 40$. Scale bar $=500 \mu \mathrm{m}$.

amounts in aggressive tumors, indicating a possible role in tumor progression. For instance, Kawada et al (29) reported that melanoma cells constitutively express CXCR3 and that its ligand, CXCL10, induces cell survival, invasion, migration, and lymph node metastasis. Goldberg-Bittman et al (30) suggested that simultaneous expression of both CXCR3 and CXCL10 in breast tumor cells indicates that CXCL10 may act not only on cells within the breast tissue microenvironment but also on the tumor cells themselves in an autocrine manner to promote tumor growth. Teichmann et al (31) raised the possibility that high endogenous CXCL10 expression in Hodgkin's lymphoma and nasopharyngeal carcinoma may not exert an antitumor effect. Mellillo et al (32) showed that, in thyroid carcinomas, overexpressed CXCL10 can promote cell proliferation and invasion.

In CRC, CXCL10 promotes invasion related properties, such as the expression of matrix-degradation enzyme, in primary as well as in metastatic sites (33). This function of CXCL10 may come into effect at primary and metastatic sites since this chemokine is actually expressed in these loci (34-38). Furthermore, Cambien et al showed that CXCR3 has emerged as an important mediator in determining the metastatic potential and site-specific spread of cancer cells, as their ligand, CXCL10, is expressed by the target organs, such as liver and lung (39).

However, little is known about the mechanism by which the circulating CXCL10 level is up-regulated in accordance with disease progression in CRC patients. In this present study, we showed that an increase in the preoperative circulating CXCL10 level was significantly correlated with factors associated with pathological $\mathrm{T}$ stage and clinical $\mathrm{H}$ stage, as defined by the Japanese Society for Cancer of the Colon and Rectum (40). There was a significant correlation between preoperative CEA and CXCL10 level. In addition, immunohistochemical analysis could detect CXCL10 expression in tumor cells from primary and metastatic sites of CRC patients, suggesting that the circulating level of CXCL10 reflects, in part, secretion from the tumor cell; therefore promoting cell proliferation and invasion in an autocrine fashion.

Regarding other sources of CXCL10 in the circulation, Cambien et al (39) demonstrated that organ-specific metastasis was induced by activation of CXCR3, facilitating the implantation and the progression of CRC cells within lung or liver tissues. Significantly higher expression of CXCL10 or CXCL11 was shown in lung or liver tissue containing CRC metastases compared to that without metastases, which suggests a possible mechanism for the promotion of the development of distant metastases in the lung or liver.

In this study we showed that CXCL10 concentration was significantly higher in patients with factors associated with venous route-metastasis (e.g., vascular invasion, distant metastasis including liver or lung metastasis). By contrast, peritoneal dissemination or factors associated with lymphatic ductal route-metastasis, such as lymphatic invasion and lymph node metastasis, were not significantly associated with CXCL10 concentration. In addition, CXCL10 was located diffusely in the cytoplasm of normal liver tissue, as well as in cancerous regions in liver metastatic tissue. These results suggested that high expression of serum CXCL10 is induced by secretion from target organs, such as liver or lung, in a paracrine fashion. This allows the revelation of the metastatic destination for CRC and allows the progression of cancer cells in these metastatic sites.

In conclusion, we identified the novel prognostic marker CXCL10 in CRC patients. Serum CXCL10 significantly correlated with vascular metastasis, such as liver or lung metastasis, but not with lymphatic metastasis. Furthermore, CXCL10 was an independent prognostic factor for overall survival. Elevated serum CXCL10 is therefore a potentially useful predictive marker for liver metastasis and overall survival in CRC. 


\section{Acknowledgments}

We thank Mrs. Motoko Ueda for providing technical assistance.

\section{References}

1. Weitz J, Koch M, Debus J, Hohler T, Galle PR and Buchler MW Colorectal cancer. Lancet 365: 153-165, 2005.

2. Andre N and Schmiegel W: Chemoradiotherapy for colorectal cancer. Gut 54: 1194-1202, 2005.

3. Lurje G, Zhang W and Lenz HJ: Molecular prognostic markers in locally advanced colon cancer. Clin Colorectal Cancer 6: 683-690, 2007.

4. Halama N, Herrmann C, Jaeger D and Herrmann T: Treatment with cetuximab, bevacizumab and irinotecan in heavily pretreated patients with metastasized colorectal cancer. Anticancer Res 28 4111-4115, 2008.

5. Fong S, Garcia Vega G and Leon V: Carcinoembryonic antigen fraction in digestive cancer. Neoplasma 32: 199-208, 1985.

6. Sugarbaker PH: Role of carcinoembryonic antigen assay in the management of cancer. Adv Immun Cancer Ther 1: 167-193, 1985.

7. Yoshie O, Imai $\mathrm{T}$ and Nomiyama $\mathrm{H}$ : Chemokines in immunity. Adv Immunol 78: 57-110, 2001.

8. Singh UP, Singh S, Iqbal N, Weaver CT, McGhee JR and Lillard JW Jr: IFN-gamma-inducible chemokines enhance adaptive immunity and colitis. J Interferon Cytokine Res 23: 591-600, 2003

9. Neville LF, Mathiak G and Bagasra O: The immunobiology of interferon-gamma inducible protein $10 \mathrm{kD}$ (IP-10): a novel, pleiotropic member of the C-X-C chemokine superfamily. Cytokine Growth Factor Rev 8: 207-219, 1997.

10. Lazzeri E and Romagnani P: CXCR3-binding chemokines: novel multifunctional therapeutic targets. Curr Drug Targets Immune Endocr Metabol Disord 5: 109-118, 2005.

11. Strieter RM, Burdick MD, Mestas J, Gomperts B, Keane MP and Belperio JA: Cancer CXC chemokine networks and tumour angiogenesis. Eur J Cancer 42: 768-778, 2006.

12. Romagnani P, Annunziato F, Lasagni L, et al: Cell cycle-dependent expression of CXC chemokine receptor 3 by endothelial cells mediates angiostatic activity. J Clin Invest 107: 53-63, 2001.

13. Narvaiza I, Mazzolini G, Barajas M, et al: Intratumoral coinjection of two adenoviruses, one encoding the chemokine IFN-gammainducible protein-10 and another encoding IL-12, results in marked antitumoral synergy. J Immunol 164: 3112-3122, 2000.

14. Mendiratta SK, Quezada A, Matar M, et al: Combination of interleukin 12 and interferon alpha gene therapy induces a synergistic antitumor response against colon and renal cell carcinoma. Hum Gene Ther 11: 1851-1862, 2000.

15. Zilocchi C, Stoppacciaro A, Chiodoni C, Parenza M, Terrazzini N and Colombo MP: Interferon gamma-independent rejection of interleukin 12-transduced carcinoma cells requires $\mathrm{CD} 4^{+} \mathrm{T}$ cells and granulocyte/macrophage colony-stimulating factor. J Exp Med 188: 133-143, 1998

16. Li G, Tian L, Hou JM, et al: Improved therapeutic effectiveness by combining recombinant $\mathrm{CXC}$ chemokine ligand 10 with cisplatin in solid tumors. Clin Cancer Res 11: 4217-4224, 2005.

17. Yao L, Pike SE, Setsuda J, et al: Effective targeting of tumor vasculature by the angiogenesis inhibitors vasostatin and interleukin-12. Blood 96: 1900-1905, 2000.

18. Proudfoot AE: Chemokine receptors: multifaceted therapeutic targets. Nat Rev Immunol 2: 106-115, 2002.

19. Romagnani P,Lasagni L, Annunziato F, Serio M and Romagnani S: CXC chemokines: the regulatory link between inflammation and angiogenesis. Trends Immunol 25: 201-209, 2004

20. Wenzel J, Worenkamper E, Freutel S, et al: Enhanced type I interferon signalling promotes Th1-biased inflammation in cutaneous lupus erythematosus. J Pathol 205: 435-442, 2005.
21. Luster AD and Leder P: IP-10, a -C-X-C- chemokine, elicits a potent thymus-dependent antitumor response in vivo. J Exp Med 178: 1057-1065, 1993.

22. Wenzel J, Bekisch B, Uerlich M, Haller O, Bieber T and Tuting T: Type I interferon-associated recruitment of cytotoxic lymphocytes: a common mechanism in regressive melanocytic lesions. Am J Clin Pathol 124: 37-48, 2005.

23. Menon AG, Janssen-van Rhijn CM, Morreau H, et al: Immune system and prognosis in colorectal cancer: a detailed immunohistochemical analysis. Lab Invest 84: 493-501, 2004.

24. Chiba T, Ohtani H, Mizoi T, et al: Intraepithelial CD8 ${ }^{+}$T-cellcount becomes a prognostic factor after a longer follow-up period in human colorectal carcinoma: possible association with suppression of micrometastasis. Br J Cancer 91: 1711-1717, 2004.

25. Pages F, Berger A, Camus M, et al: Effector memory T cells, early metastasis, and survival in colorectal cancer. N Engl J Med 353: 2654-2666, 2005.

26. Sato E, Fujimoto J, Toyoki H, et al: Expression of IP-10 related to angiogenesis in uterine cervical cancers. Br J Cancer 96: 1735-1739, 2007.

27. Jiang Z, Xu Y and Cai S: CXCL10 expression and prognostic significance in stage II and III colorectal cancer. Mol Biol Rep 37: 3029-3036, 2010

28. Ben-Baruch A: The multifaceted roles of chemokines in malignancy. Cancer Metastasis Rev 25: 357-371, 2006

29. Kawada K, Sonoshita M, Sakashita H, et al: Pivotal role of CXCR3 in melanoma cell metastasis to lymph nodes. Cancer Res 64: 4010-4017, 2004

30. Goldberg-Bittman L, Neumark E, Sagi-Assif O, et al: The expression of the chemokine receptor CXCR 3 and its ligand, CXCL10, in human breast adenocarcinoma cell lines. Immunol Lett 92: 171-178, 2004.

31. Teichmann M, Meyer B, Beck A and Niedobitek G: Expression of the interferon-inducible chemokine IP-10 (CXCL10), a chemokine with proposed anti-neoplastic functions, in Hodgkin lymphoma and nasopharyngeal carcinoma. J Pathol 206: 68-75, 2005.

32. Melillo RM, Castellone MD, Guarino V, et al: The RET/PTCRAS-BRAF linear signaling cascade mediates the motile and mitogenic phenotype of thyroid cancer cells. J Clin Invest 115: 1068-1081,2005

33. Zipin-Roitman A, Meshel T, Sagi-Assif O, et al: CXCL10 promotes invasion-related properties in human colorectal carcinoma cells. Cancer Res 67: 3396-3405, 2007.

34. Wang H, Gao X, Fukumoto S, Tademoto S, Sato K and Hirai K: Differential expression and regulation of chemokines JE, KC, and IP-10 gene in primary cultured murine hepatocytes. J Cell Physiol 181: 361-370, 1999.

35. Patzwahl R, Meier V, Ramadori G and Mihm S: Enhanced expression of interferon-regulated genes in the liver of patients with chronic hepatitis $C$ virus infection: detection by suppressionsubtractive hybridization. J Virol 75: 1332-1338, 2001.

36. Ren X, Kennedy A and Colletti LM: CXC chemokine expression after stimulation with interferon-gamma in primary rat hepatocytes in culture. Shock 17: 513-520, 2002.

37. Barsig J, Flesch IE and Kaufmann SH: Macrophages and hepatocytic cells as chemokine producers in murine listeriosis. Immunobiology 199: 87-104, 1998.

38. Dwinell MB, Lugering N, Eckmann L and Kagnoff MF: Regulated production of interferon-inducible T-cell chemoattractants by human intestinal epithelial cells. Gastroenterology 120: 49-59, 2001.

39. Cambien B, Karimdjee BF, Richard-Fiardo P, et al: Organspecific inhibition of metastatic colon carcinoma by CXCR3 antagonism. Br J Cancer 100: 1755-1764, 2009.

40. Japanese Classification of Colorectal Carcinoma SEE, Japanese Society for Cancer of the Colon and Rectum, Kanehara \& Co., Ltd., Tokyo, Japan. 